\title{
Lipid MALDI MS Profiles of Gastric Cancer
}

\author{
Sun Young Kwon ${ }^{1}$, Seung Ho Choi ${ }^{2}$, Young Soo Park ${ }^{3}$, Do Youn Park ${ }^{4}$, Young Iee Park ${ }^{2}$, Ilseon \\ Hwang $^{1}$, Min Hee Ryu ${ }^{3}$, Chae Hwa Kwon ${ }^{4}$, Jeong Hwa Lee ${ }^{5}$, Geul Bang ${ }^{6}$, Kwang Pyo Kim ${ }^{5}$, Young \\ Hwan $\mathrm{Kim}^{6}$ and Hark Kyun $\mathrm{Kim}^{2, *}$
}

${ }^{1}$ Keimyung University Dongsan Hospital, Daegu, Korea

${ }^{2}$ National Cancer Center, Goyang, Korea

${ }^{3}$ Asan Medical Center, Seoul, Korea

${ }^{4}$ Pusan National University Hospital, Pusan, Korea

${ }^{5}$ Department of Molecular Biotechnology, Konkuk University, Seoul, Korea

${ }^{6}$ Division of Mass Spectrometry Research, Korea Basic Science Institute, Ochang, Korea

\begin{abstract}
Tissue matrix-assisted laser desorption/ionization mass spectrometry (MALDI MS)may identify lipids differentially expressed between cancer and adjacent normal tissue. To identify lipidomic profiles for gastric cancer, 24 gastric cancerswere profiled for lipid by the histology-directed, tissue MALDI MS technology. Lipid profiles differed between gastric cancer and adjacent normal tissue samples. At $P<0.05$, median class prediction accuracy in 100 random training-to-test partitions was $83.3 \%(5 / 6)$ for all classifiers tested. A peak at m/z 741.6 (sphingomyelin 34:1 K) was overexpressed, and a peak at m/z 782.6 (phosphatidylcholine 34:1 Na) was underexpressed in gastric cancers compared with normal tissue. Thus, lipid MALDI MS analysis may capture a global alteration in lipid profile of gastric cancer tissue, distinguishing cancerous epithelium from normal epithelium.
\end{abstract}

Keywords: Gastric cancer, lipid, MALDI, profile.

\section{INTRODUCTION}

Gastric cancer is the second most common cause of cancer death, and the incidence of diffuse-type gastric cancer has not been decreasing in Western countries [1]. There are no tissue-specific biomarkers that can be used for immunohistochemical diagnosis of gastric cancers. Tumor markers, such as CEA, CA19-9, and CA72-4, are not useful for the gastric cancer screening because of low sensitivity and specificity [2]. Clearly, there is an unmet need for sensitive and specific diagnostic markers for gastric cancer, especially early-onset gastric cancers [3].

Protein and lipid profiles obtained from histologydirected, tissue matrix-assisted laser desorption/ionization mass spectrometry (MALDIMS) are demonstrated to accurately differentiate cancer tissue from adjacent normal tissue[4]. Using 2,5-dihydroxybenzoic acid (DHB) and $\alpha$ cyano-4- hydroxycinnamic acid (CHCA) matrix, our group identified lipid profiles for several common cancers $[5,6]$. We initiated a study to evaluate whether histology-directed, lipid MALDI MS analysis may assist with the diagnosis of gastric cancers.

\footnotetext{
*Address correspondence to these authors at the Biomolecular Function Research Branch, Research Institute, National Cancer Center, 323 Ilsanro, Ilsan, Goyang, Gyeonggi, 410-769, Republic of Korea;

Tel: +82-31-920-2238; Fax: +82-31-920-2006; E-mail: hkim@ncc.re.kr
}

\section{MATERIALS AND METHODS}

\section{Acquisition and Processing of MALDI MS Data}

This study was performed on surgical samples collected from young gastric cancer patients undergoing surgery at several participating hospitals in Korea from 2008 to 2012. All patients signed informed consents. Basically, histologydirected MADLI MS analysis methods, pioneered by Dr. Caprioli, were used for this study [reviewed in [7]]. $10 \mu \mathrm{m}$ thick cryosection slides were obtained from each frozen tissue. One section slide was stained with hematoxylin and eosin (H\&E). Next tissue sections were thaw-mounted to indium tin oxide (ITO) slides(HST Inc., Newark, NJ) and dried in vacuum. 2,5-dihydroxybenzoic acid (DHB) and $\alpha$ cyano-4-hydroxycinnamic acid (CHCA) was dissolved in $70 \%$ methanol plus $0.1 \%$ trifluoroacetic acid and $1 \%$ piperidine [5]. This matrix solution was deposited on cryosection slides by the Chip-1000 instrument (Shimadzu, Kyoto, Japan) [8]. Interval between matrix spots was 400 $\mu \mathrm{m}$. Mass spectra were acquired using UltrafleXtreme (Bruker Daltonics, Bremen, Germany) at a laser frequency of $1,000 \mathrm{~Hz}$. Calibration standards were as follows; $\mathrm{m} / \mathrm{z} 674$ 834 (positive ion mode) and $\mathrm{m} / \mathrm{z}$ 564-906 (negative ion mode). Mass spectra originating from tumor (or glandular epithelium)-rich spots only were acquired for subsequent processing (Fig 1A). ClinProTools(v 2.2, Bruker Daltonics) was used for peak processing. 


\section{Statistical Analysis}

Average-normalized datasets of each of positive- and negative-ion modes were combined. Bioinformatic analyses were performed using BRB-ArrayTools (version 4.1, Biometrics Branch, US National Cancer Institute) [9]. To assess the predictive power of discriminatory peaks, we performed class prediction analyses by randomly dividing the whole dataset into training and test subsets with 1-to-1 ratio, using statistical analysis methods previously described [10]. For each random dataset, predictors identified in each training set were used to predict the class label of samples in the test set. To assign the molecular identify of informative peaks, MALDI MS/MS analysis was performed on cryosections, and the data were mapped to public lipid databases (www.lipidmaps.org).

\section{RESULTS}

Histology-directed, lipid MALDI MS analyses were performed for 24 retrospective, frozen surgical tissue samples (12 pairs of cancer and adjacent normal tissue samples) (Table 1). There were 7 females (58.3\%) and median age was 35 years [3]. Six patients $(50 \%)$ had signet ring cell carcinomas. The average MALDI MS spectra were composed of median 5 individual measurements for cancer samples and median 4 individual measurements for normal samples. The average mass spectra for gastric cancers and
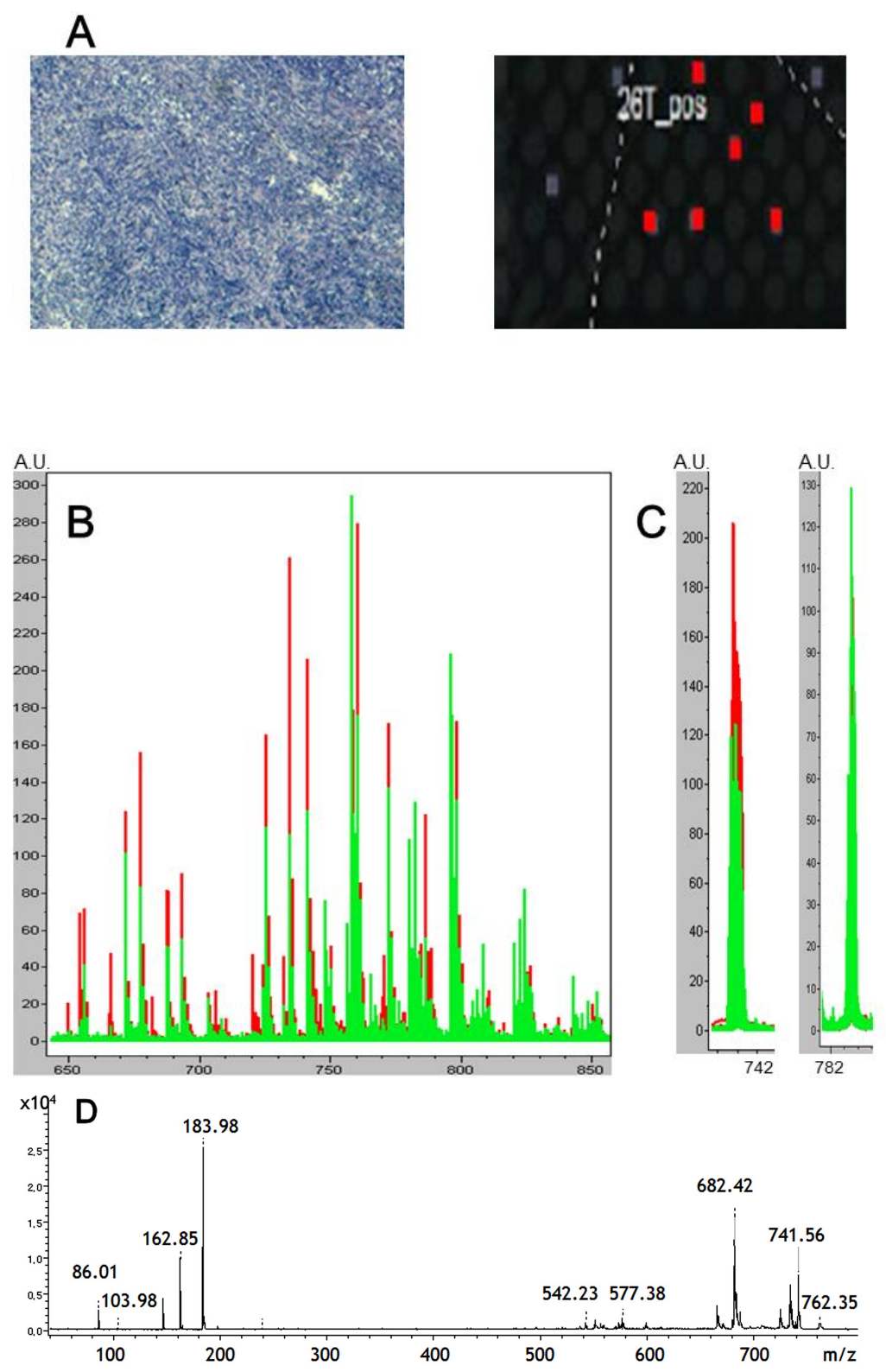

Fig. (1). A. An optical image of matrix-loaded spots on an ITO cryosection slide, and a corresponding H\&E section for a representative gastric cancer sample (x 40 magnification). Mass spectra originating from tumor-rich spots (red) only were processed for subsequent statistical analyses. B. Overlays of average mass spectra obtained from gastric cancer samples (red) and adjacent normal tissue samples (green) in the positive ion mode [10]. C. Intensity profiles of SM 34:1 (m/z 741.6, left) and PC 34:1 (m/z 782.6, right) in gastric cancers (red) and normal tissue samples (green). D. The MS/MS profile for a peak at m/z 741.6, which was assigned as SM 34:1 [M+K]+. 
Table 1. Peaks Differentially Expressed Between Gastric Cancers and Normal Samples at Feature Selection $p<0.05$.

\begin{tabular}{|c|c|c|c|}
\hline \multicolumn{4}{|c|}{ Increased in Cancer } \\
\hline $\mathbf{m} / \mathbf{z}$ & $\mathbf{P}$ & Ratio $^{1}$ & Assignment \\
\hline P741.6 & 0.002 & 1.3 & $\mathrm{SM} 34: 1[\mathrm{M}+\mathrm{K}]^{+}$ \\
\hline P693.62 & 0.030 & 1.2 & \\
\hline P786.64 & 0.036 & 1.2 & PC 33:0 $[\mathrm{M}+\mathrm{K}]^{+}$ \\
\hline \multicolumn{4}{|c|}{ Decreased in Cancer } \\
\hline $\mathbf{m} / \mathbf{z}$ & $\mathbf{p}$ & Ratio & Assignment \\
\hline P782.6 & $<0.001$ & 0.7 & PC $34: 1[\mathrm{M}+\mathrm{Na}]^{+}$ \\
\hline P636.0 & $<0.001$ & 0.7 & \\
\hline P804.6 & 0.006 & 0.8 & \\
\hline
\end{tabular}

Ratio $^{1}$, tumor/normal; P, positive ion mode; SM, sphingomyelin; PC, phosphatidylcholine

adjacent normal tissue are shown in the left panel of Fig. (1B). Finally, 74 processed peaks (28 and 46 for positive and negative modes, respectively) were analyzed.

Twelve pairs of gastric cancer and adjacent normal tissue samples were compared using paired $t$-test. Table 1 lists 6 peaks were differentially expressed between cancer and normal samples at feature selection $p<0.05$ (Fig 1C). Permutation $P$ value for cross-validated misclassification rate was at borderline significance, when all of the 12 pairs were used as a training set (the compound covariate predictor: 0.03 , diagonal linear discriminant analysis: 0.01 , 1-and 3-nearest neighbors: 0.08 and 0.1 , nearest centroid: 0.1 , and the support vector machine: 0.04). These results suggest that cancer and normal samples are different in lipid profiles. To assess the predictive power of discriminatory peaks, we performed class prediction analyses by randomly dividing the whole dataset into training and test subsets with 1-to-1 ratio [10]. At $P<0.05$, median class prediction accuracy in 100 random training-to-test partitions was $83.3 \%$ $(5 / 6)$ for all classifiers tested. Thus, lipid profiles of gastric cancers are different from those of adjacent normal tissue.

Using MS/MS analysis, peaks overexpressed in gastric cancerswere identified for the exact molecular assignment (Table 1). Peaks at $\mathrm{m} / \mathrm{z} 741.6, \mathrm{~m} / \mathrm{z} 786.6$, and $\mathrm{m} / \mathrm{z} 782.6$ in the positive ion mode were identified as SM 34:1 $[\mathrm{M}+\mathrm{K}]^{+}$, phos-phatidylcholine (PC) 33:0 $[\mathrm{M}+\mathrm{K}]^{+}$, and PC $34: 1$ $[\mathrm{M}+\mathrm{Na}]^{+}$, respectively (Fig. 1D).

\section{DISCUSSION}

This study compares lipid profiles between gastric cancers and adjacent normal tissue. There is an urgent, unmet need for novel markers for this aggressive disease [3]. Advantages of histology-directed MALDI MS technology include rapid procedure time, low reagent cost, and small amount of tissue required. According to the MALDI MS analyses of gastric cancer samples, sphingomyelin 34:1 was identified to be the most significantly overexpressed lipid. In addition, PC 34:1 $[\mathrm{M}+\mathrm{Na}]^{+}(\mathrm{m} / \mathrm{z} 782.6)$, which is over expressed in lung cancers and cholangiocarcinomas $[5,6]$, was underexpressed in gastric cancer patients, while PC 33:0
$[\mathrm{M}+\mathrm{K}]^{+}$was overexpressed. Future larger-scale studies will be needed to validate our findings.

\section{CONFLICT OF INTEREST}

The authors confirm that this article content has no conflict of interest.

\section{ACKNOWLEDGEMENTS}

The work was supported by 2013 K00429 and the Proteogenomic Research Program through the National Research Foundation of Korea funded by the Korean Ministry of Education, Science and Technology.

\section{ABBREVIATIONS}

$\begin{array}{lll}\text { MALDI MS } & =\begin{array}{l}\text { Matrix-assisted laser desorption } \\ \text { ioniziation mass spectrometry }\end{array} \\ \text { DHB } & =2,5 \text {-dihydroxybenzoic acid } \\ \text { CHCA } & =\alpha \text {-cyano-4- hydroxycinnamic acid } \\ \text { ITO } & =\text { Indium tin oxide } \\ \text { SM } & =\text { Sphingomyelin } \\ \text { PC } & =\text { Phosphatidylcholine }\end{array}$

\section{REFERENCES}

[1] Alberts, S.; Cervantes, A.; van de Velde, C. Gastric cancer: epidemiology, pathology, and treatment. Ann. Oncol., 2003, 14, 31-6.

[2] Shimada, H.; Noie, T.; Ohashi, M.; Oba, K.; Takahashi, Y. Clinical significance of serum tumor markers for gastric cancer: a systematic review of literature by the Task Force of the Japanese Gastric Cancer Association. Gastric Cancer, 2013 Apr 10. [Epub ahead of print]

[3] Smith, B.R.; Stabile, B.E. Extreme aggressiveness and lethality of gastric adenocarcinoma in the very young. Arch. Surg., 2009, 144(6), 506-10

[4] Kim, H.K.; Reyzer, M.L.; Choi, I.J.; Kim, C.G.; Kim, H.S.; Oshima, A.; Chertov, O.; Colantonio, S.; Fisher, R.J.; Allen, J.L.; Caprioli, R.M.; Green, J.E. Gastric cancer-specific protein profile identified using endoscopic biopsy samples via MALDI mass spectrometry. J. Proteome Res., 2010, 9 (8), 4123-30.

[5] Lee, G.K.; Lee, H.S.; Park, Y.S.; Lee, J.H.; Lee, S.C.; Lee, J.H.; Lee, S.J.; Shanta, S.R.; Park, H.M.; Kim, H.R.; Kim, I.H.; Kim, 
Y.H.; Zo, J.I.; Kim, K.P.; Kim, H.K. Lipid MALDI profile classifies non-small cell lung cancers according to the histologic type. Lung Cancer, 2012, 76 (2), 197-203.

[6] Park, Y.S.; Yoo, C.W.; Lee, S.C.; Park, S.J.; Oh, J.H.; Yoo, B.C.; Paik, S.S.; Lee, K.G.; Jin, S.Y.; Kim, S.C.; Kim, K.P.; Kim, Y.H.; Choi, D; Kim, H.K. Lipid profiles for intrahepatic cholangiocarcinoma identified using matrix-assisted laser desorption/ionization mass spectrometry. Clin. Chim. Acta., 2011, 412 (21-22), 1978-82.

[7] Seeley, E.H.; Caprioli, R.M. MALDI $3^{\text {rd }}$ imaging mass spectrometryn of human tissue: method challenges and clinical perspectives. Trends Biotechnol., 2011, 29 (3), 136-43.
[8] Kim, Y.; Shanta, S.R.; Zhou, L.H.; Kim, K.P. Mass spectrometry based cellular phosphoinositides profiling and phospholipid analysis: a brief review. Exp. Mol. Med., 2010, 42 (1), 1-11.

[9] Simon, R.; Lam, A.; Li, M.C.; Ngan, M.; Menenzes, S.; Zhao, Y. Analysis of gene expression data using BRB-ArrayTools. Cancer Inform., 2007, 3, 11-7.

[10] Kang, H.S.; Lee, S.C.; Park, Y.S.; Jeon, Y.E.; Lee, J.H.; Jung, S.Y.; Jang, S.H.; Park, H.M.; Yoo, C.W.; Park, S.H.; Han, S.Y.; Kim, K.P.; Kim, Y.H.; Ro, J.; Kim, H.K. Protein and lipid MALDI profiles classify breast cancers according to the intrinsic subtype. BMC Cancer, 2011, 11, 465 .

(C) Kwon et al.; Licensee Bentham Open.

This is an open access article licensed under the terms of the Creative Commons Attribution Non-Commercial License (http://creativecommons.org/licenses/ by-nc/3.0/) which permits unrestricted, non-commercial use, distribution and reproduction in any medium, provided the work is properly cited. 